\title{
Arthroscopic techniques and instruments for meniscal allograft transplantation using the bone bridge in trough method
}

\author{
YADONG ZHANG, SHUXUN HOU, LI LI, HONGBIN ZHONG, YICHAO ZHANG, \\ DIANZHONG LUO, LEI CHEN, YUNPENG DING, DONGQIANG GU and HAIGANG JIA
}

Department of Orthopedics, The First Affiliated Hospital of PLA General Hospital (304 Hospital), Beijing 100048, P.R. China

Received June 20, 2018; Accepted October 26, 2018

DOI: $10.3892 /$ etm.2018.7090

\begin{abstract}
The aim of the current study was to investigate the construction of the bone bridge and tibial plateau under arthroscopy during meniscal allograft transplantation, in order to simplify and enhance the accuracy of bone bridge fixation intraoperatively. A traction line passed through the attachment of the anterior and posterior horns of the superior meniscus to the bone bridge was used to pull the bone bridge into the knee joint cavity and fix the anteroposterior horns of the meniscus. At the junction of the body of the meniscus and the posterior and anterior horns of the meniscus, a traction line was created at the anterior and posterior $1 / 3$ of the meniscus to pull and fix the meniscus. Under the arthroscope, the aiming device was placed on the tibial plateau. The direction and width of the guide plate were identical to those of the bone trough of the tibial plateau. The bone tunnel was made using the guide needle and a 9-mm hollow drill, the piston rod was inserted, and the arch-shaped bone knife was inserted along with the piston rod to construct the 9-mm bone trough of the tibial plateau. The periphery of the meniscus was sutured to the joint capsule. These surgical techniques and instruments could standardize meniscal graft transplantation and avoid the incidence of surgical errors caused by mismatched size and shape of the bone bridge and bone trough. This would make the surgery more convenient, safe and accurate. The four-point fixation of the tibial plateau contributed to preventing the reversal of the meniscus during transplantation, and partially reconstructed the coronary ligament of the meniscal tibia, which probably enhanced the stability of the meniscus and minimized the risk of extrusion of the meniscal allograft. The bone bridge and bone trough of the tibial plateau were properly constructed under arthroscopy. Dynamic monitoring of surgical indications, explicit preoperative preparation and
\end{abstract}

Correspondence to: Dr Yadong Zhang, Department of Orthopedics, The First Affiliated Hospital of PLA General Hospital (304 Hospital), 51 Fucheng Road, Beijing 100048, P.R. China E-mail: zhangyadong2018@sina.com

Key words: menisci, meniscal allograft transplantation, arthroscopy, minimally invasive surgery, surgical instrument standardized surgical procedures could achieve high efficacy and excellent fixation effect during meniscal graft transplantation. The four-point fixation of the tibial plateau maintains and enhances the stability of the meniscal allograft, reduces the risk of meniscal extrusion and ensures the postoperative recovery of meniscal function.

\section{Introduction}

Meniscal allograft transplantation is widely applied in the reconstruction of the meniscus (1-5), which consists of open surgery and arthroscopic minimally invasive surgery. Although the results of open and arthroscopic minimally invasive surgery are similar $(6,7)$, arthroscopic minimally invasive surgery can obtain a comprehensive and clear intra-articular visual field, which facilitates the treatment of concomitant joint lesions, reduces surgical injury, and lowers the risk of postoperative tissue adhesions. Due to the lack of standard instruments and technical specifications, it is not possible to perform all meniscal allograft transplantation procedures by arthroscopic surgery, and merely open surgery or arthroscope-assisted small incision surgical procedures may be performed instead (8).

The quality of the fixation of the meniscal anteroposterior horns determines the effectiveness of meniscal allograft transplantation (9). It is simpler to directly suture and fix the anterior and posterior horns of the meniscus, but fixation of the anterior and posterior horns of the meniscus with attached bone anchors possesses significant biomechanical advantages (10-12), and serves as the gold standard for meniscal allograft transplantation (13). Fixation using attached bone anchors involves two processes. First, the anterior and posterior horns of the meniscus are made into two bone plugs, and implanted into respective bone tunnels of the tibial plateau. Second, a bone bridge connecting the anterior and posterior horns of the meniscus is maintained and implanted into the bone trough between the anterior and posterior horns of the tibial plateau.

The former process only induces slight trauma to the tibial plateau, but it is technically demanding. It may lead to inaccurate distance and position between the anterior and posterior horns, thereby affecting the function of the transplanted meniscus (14). It is not recommended for application in lateral meniscal transplantation. Due to the short distance between 
the anterior and posterior horns, the risk of inter-junction of the two bone tunnels of the tibial plateau is relatively high. The latter process can ensure that the position of the anterior and posterior horns of the meniscus is almost normal, and maintain a normal distance and anatomical position between the anterior and posterior horns of the meniscus (14). The standardization of surgical procedures can reduce the risk of improper positioning of the meniscus, and properly reconstruct the function of the meniscus in buffering annular stress (1).

The standardization of surgical procedures can be applied in medial or lateral meniscus transplantation, particularly the latter. However, the process is disadvantaged by a lack of standard minimally invasive surgical instruments, and surgeons can only rely on manual repair and grinding under visual inspection. It is difficult to ensure that the shape and size of the bone trough is properly matched with the bone bridge, which requires repeated comparison, modification and grinding. In addition, due to the absence of technical standards $(8,15,16)$, it is difficult to maintain optimal stability of the anterior and posterior horns of the meniscus. The construction of the bone tunnel and bone bridge is time consuming, which increases surgical risk.

To resolve the technical issues of bone bridge placement in meniscal allograft transplantation, a minimally-invasive surgical technique and corresponding surgical instruments for arthroscopic meniscus transplantation was established in the current study to standardize the construction of the anterior and posterior horn bone bridges of the meniscus and properly match with the bone tunnels of the tibial plateau. The aim of this novel technique was to accurately and reliably accomplish meniscal allograft transplantation, enhance the compatibility and stability of the bone bridge and bone tunnel and improve the surgical effect.

\section{Materials and methods}

Ethical approval. The current study was approved by the Ethics Committee of the First Affiliated Hospital of PLA General Hospital (304 Hospital; Beijing, China). Written informed consent was obtained from all participants. Participants were recruited from November 2006 to July 2009. In total, 21 patients (male, $n=14$ and female, $n=7$; age range, 19-49 years; mean age, 31.6 years) with meniscal resection underwent allogeneic meniscus transplantation.

Surgical instruments. Surgical instruments included two sets of bone bridge instruments and bone trough instruments. The bone bridge instruments (Fig. 1) consisted of a fixation table and a self-made bone knife. The meniscus fixation table (Fig. 1A) was utilized to hold and fix the meniscal allograft. The standard-sized meniscus bone bridge cutting mold was equipped, and the self-made bone knife (Fig. 1B) was used to cut the standard-sized meniscal bone bridge. The bone trough instruments (Fig. 2) consisted of an aiming device (Fig. 2A), guide needle obturator (Fig. 2B), guide needle, standard-sized hollow drill (Fig. 2C), bone trough and standard-sized trough bone knife (Fig. 2D).

Construction of the allogenic meniscal bone bridge. The meniscal allograft was soaked and thawed. The excess peripheral synovial tissues were removed and the redundant lateral bone was eliminated. The allogenic meniscus was reversed and placed on a fixation table, so that the anterior and posterior horns of the meniscus were resected to the edges of the bone knife. The bone anchors between the anterior and posterior horns of the meniscus were fixed at four different points. The sagittal direction of the bone anchors was close to the bone knife, and the other end was adjacent to the anvil (Fig. 3). The bone anchors were cut with a self-made trough bone knife $(8.5 \mathrm{~mm}$ in diameter) to construct the standard-sized bone bridge (Fig. 4). The bone bridge between the anterior and posterior horns and the allogenic meniscus were removed from the fixation table. The bone bridge was then slightly modified to make it more regular and smoother to adapt to the length of the bone trough of the tibial plateau. Non-absorbable wire [2-0 Ticron (Medtronic, Inc., NJ, USA) or Ethibond (Ethicon, Somerville, NJ, USA)] was inserted at the attachment of the anterior and posterior horns of the meniscus of the bone bridge to allow the bone bridge to enter into the bone trough and fix the anterior and posterior horns of the meniscus. The anterior and posterior $1 / 3$ of the traction wire of the meniscus was inserted at the junction of the meniscal body and the posterior horn, and the meniscal body and the anterior horn. Absorbable sutures were used to pull and fix the meniscus, and the surface of the meniscus was stained with methylene blue (Fig. 5).

Surgical position and incision. The affected limb was suspended above the operating table with $90^{\circ}$ of knee flexion or the patients were placed in a supine position with the affected limb placed on the operating table in the '4' shape. Arthroscopic transverse medial and anterolateral incisions were created. Because both the instrument manipulation and the implantation of allogenic meniscus passed through the medial incision, the anterior medial incision was cut slightly longer $(\sim 1.5 \mathrm{~cm})$. If necessary, a short arc incision with the apex toward the ankle was made, or a longitudinal incision in a zig-zag shape was created in the direction of the ankle. A lateral incision could be made to protect the posterior lateral knee of the common peroneal nerve above the humeral head.

Positioning of the aiming device and bone trough of the tibial plateau. The bone trough was made on the tibial plateau. The guide plate of the aiming device was arthroscopically inserted through the medial incision into the knee joint space. The knee joint was slightly straightened to facilitate adjustment of the guide plate of the aiming device. The guide plate of the aiming device was close to the lateral or medial edge of the tibial plateau of the anterior cruciate ligament, and the guide plate was aligned with the position, width and direction of the bone trough of the tibial plateau. The surgeons could adjust and predict the position, width and direction of the bone trough through the guide plate. The sagittal direction of the guide plate was parallel to the sagittal axis of the tibial plateau, and the horizontal direction was parallel to the tibial articular surface (Fig. 6). A C-arm fluoroscope was utilized to determine the position and direction of the guide plate, and to determine the position, direction and depth of the guide needle during needle insertion (Fig. 7A). 
Table I. Comparison of the evaluation scores of knee joint function before surgery and at final follow-up.

\begin{tabular}{|c|c|c|c|c|c|c|}
\hline \multirow[b]{2}{*}{ Time } & \multirow[b]{2}{*}{ VAS score } & \multirow[b]{2}{*}{ Lysholm score } & \multicolumn{4}{|c|}{ IKDC classification } \\
\hline & & & A & B & $\mathrm{C}$ & $\mathrm{D}$ \\
\hline Before operation & $7.0 \pm 2.3$ & $64.3 \pm 15.7$ & & 3 & 11 & 7 \\
\hline Final follow-up & $3.2 \pm 1.3$ & $80.1 \pm 19.4$ & 6 & 10 & 3 & 2 \\
\hline Statistical analysis & $\mathrm{t}=6.591 ; \mathrm{P}=0.000$ & $\mathrm{t}=2.687 ; \mathrm{P}=0.011$ & $\begin{aligned} \mathrm{Hc} & =21.159 \\
\mathrm{P} & =0.0003\end{aligned}$ & & & \\
\hline
\end{tabular}

VAS, visual analog scale; IKDC, International Knee Documentation Committee.
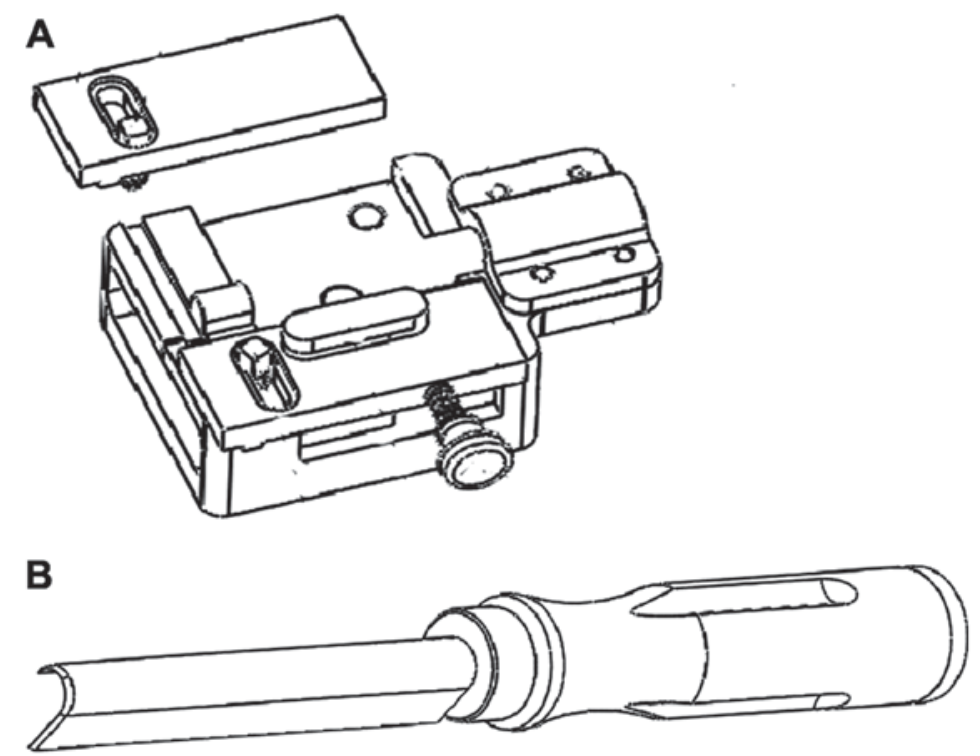

Figure 1. Schematic diagram of a meniscal bone graft device. (A) Meniscus fixation table. (B) Self-made bone knife.

Insertion of the guide wire into the bone trough. The top of the guide plate was attached to the posterior edge of the tibial plateau. The top and bottom plates of the guide plate were tightly attached to the anterior and posterior margins of the tibial plateau to fix the position of the guide plate. The needle obturator of the aiming device was inserted into the cannula below the guide plate and a 2 -mm guide wire was inserted along with the center of the guide needle obturator until the needle contacted with the guide plate. According to the scale on the guide needle, the length of guide needle insertion was recorded. C-arm fluoroscopy was performed to verify if the guide needle was properly inserted (Fig. 7B).

Construction of bone trough of tibial plateau. After the guide needle was inserted, the guide needle obturator was removed, and a 9-mm hollow drill was inserted along with the guide needle. The length of the hollow drill was $5 \mathrm{~mm}$ shorter than that of the guide needle, and the aiming device, guide plate and hollow drill were removed. A 7-mm piston rod was placed in the $9-\mathrm{mm}$ bone tunnel. The length between the anterior and posterior edges of the bone tunnel was measured according to the scale on the piston rod, which was the length of the bone trough of the tibial plateau. Using a semicircular arched bone knife with an outer diameter of $9 \mathrm{~mm}$, the bone fragments

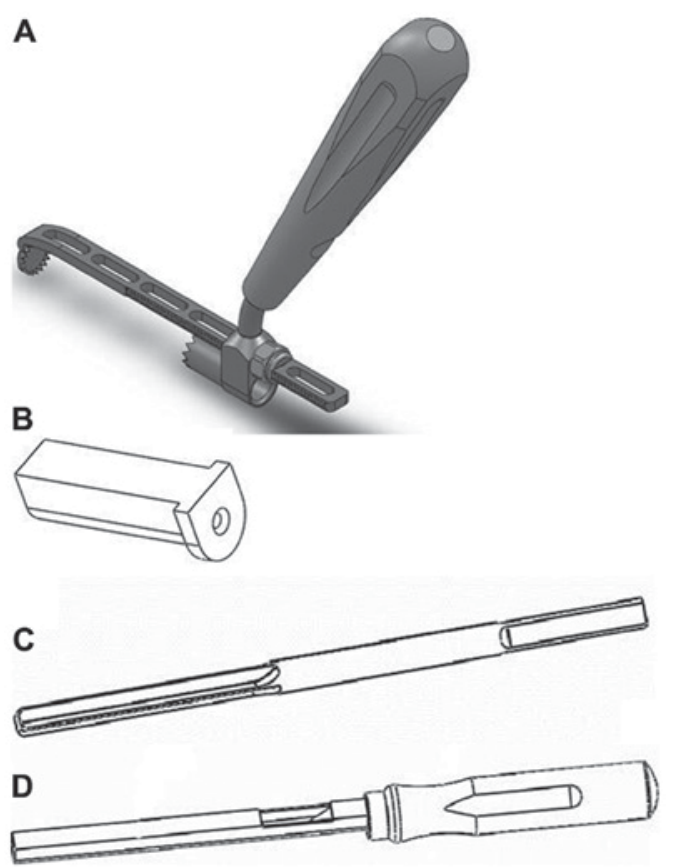

Figure 2. Schematic diagram of a meniscal bone graft instrument (A) Aiming device. (B) Guide needle obturator. (C) Standard-sized hollow drill. (D) Trough bone knife. 


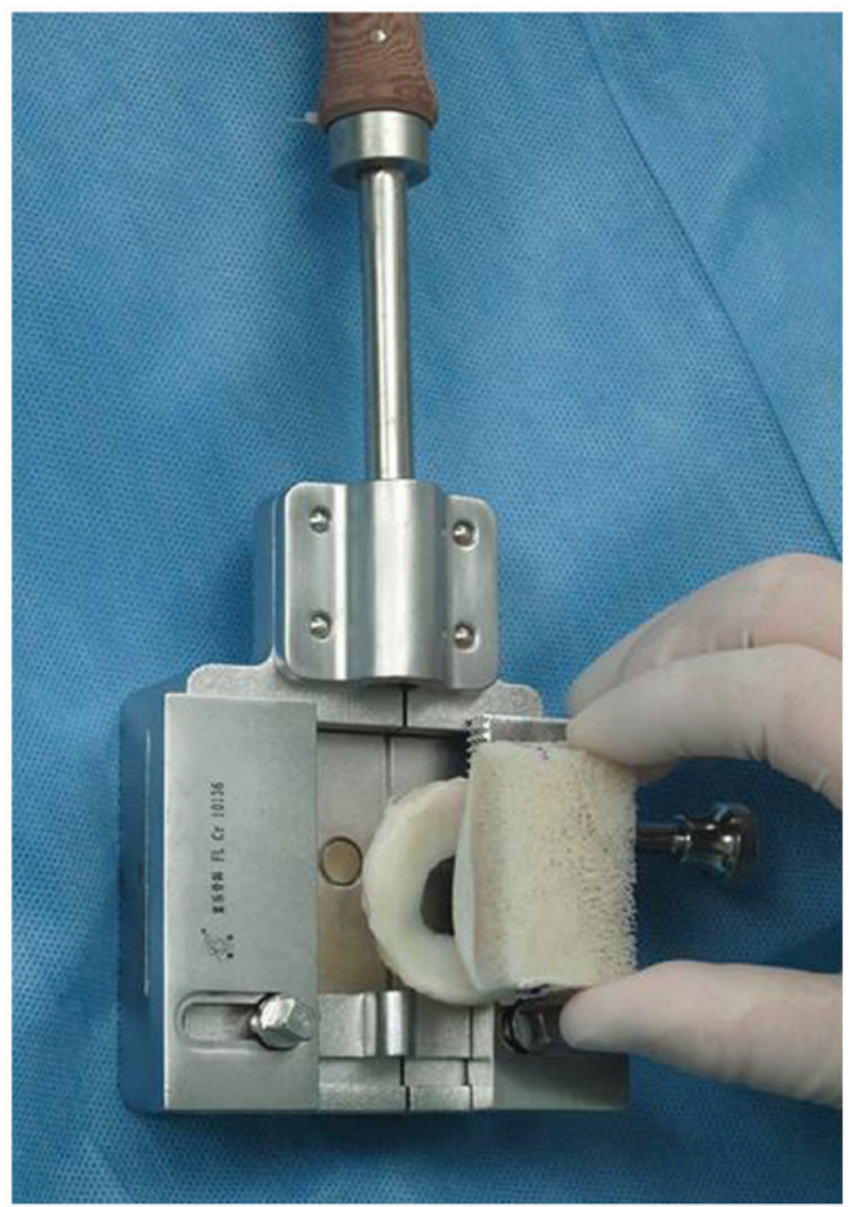

Figure 3. Positioning of the meniscus allograft on the fixation table. The meniscus allograft was reversed and placed on a fixation table, so that the anterior and posterior horns of the meniscus were resected to the edges of the bone knife. The bone anchors between the anterior and posterior horns of the meniscus were fixed at four different points. The sagittal direction of the bone anchors was close to the bone knife, and the other end was adjacent to the anvil.

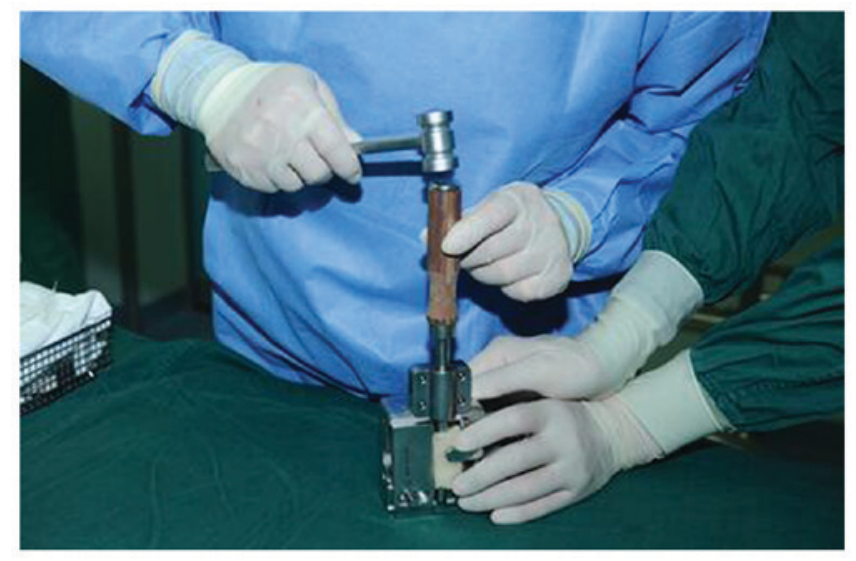

Figure 4. Construction of the bone bridge. The bone fragments were cut using a self-made trough bone knife $(8.5 \mathrm{~mm}$ in diameter) and the standard-sized bone bridge was constructed.

were eliminated, the bone trough was modified, and the bone trough of the tibial plateau with a 9-mm diameter and width was constructed (Fig. 8).

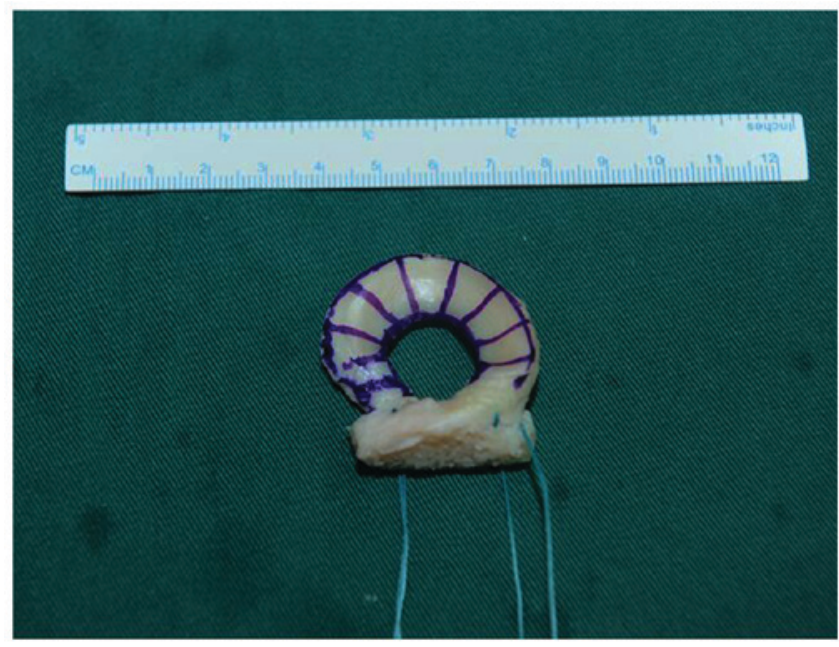

Figure 5. Attachment of the meniscus to the bone bridge. The meniscal allograft was removed from the fixation table. Non-absorbable traction wire was inserted at the attachment of the anterior and posterior horns of the meniscus of the bone bridge to pull the bone bridge into the bone trough and fix the anteroposterior horn of the meniscus. The surface of the variant meniscus was stained with methylene blue.

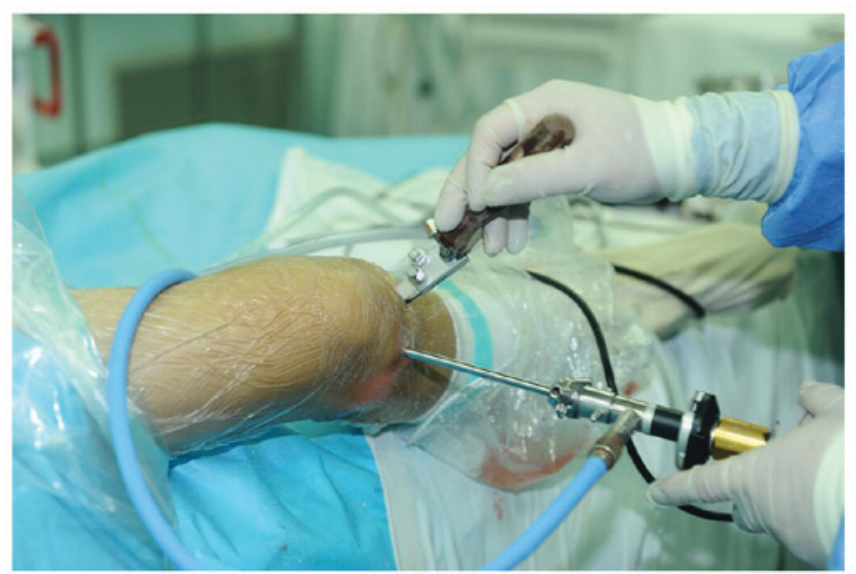

Figure 6. Positioning of the aiming device and bone trough of the tibial plateau. Under arthroscopic guidance, the guide plate of the aiming device was inserted into the gap of the knee joint through the medial incision. The aiming device was placed on the lateral or medial tibial plateau according to the meniscus, and the guide plate was close to the lateral or medial edge of the tibial plateau of the anterior cruciate ligament from the center of the anterior horn to the center of the posterior horn of the meniscus. The sagittal orientation was parallel to the sagittal axis of the tibial plateau, and the horizontal orientation was parallel to the tibial articular surface.

Construction of bone tunnel of anterior and posterior horns of meniscus. A 2-cm longitudinal incision was made in the medial side of the tibial tuberosity, and a bone hole was created from the anterior medial side of the tibia to the bottom of the bone trough of the tibial plateau. The traction line in the anterior and posterior horns of the meniscus passed the anterior medial tibia through the bone hole. It was necessary to ensure that the distance between the bone tunnel of the bone trough and the suture of the bone bridge, as well as the anterior horn of the original meniscus was equivalent. At the junction of the meniscal body and the posterior horn and the junction of the meniscus body and the anterior horn, the anterior and posterior $1 / 3$ of the traction line was pulled outside the skin via the joint (Fig. 9A). 


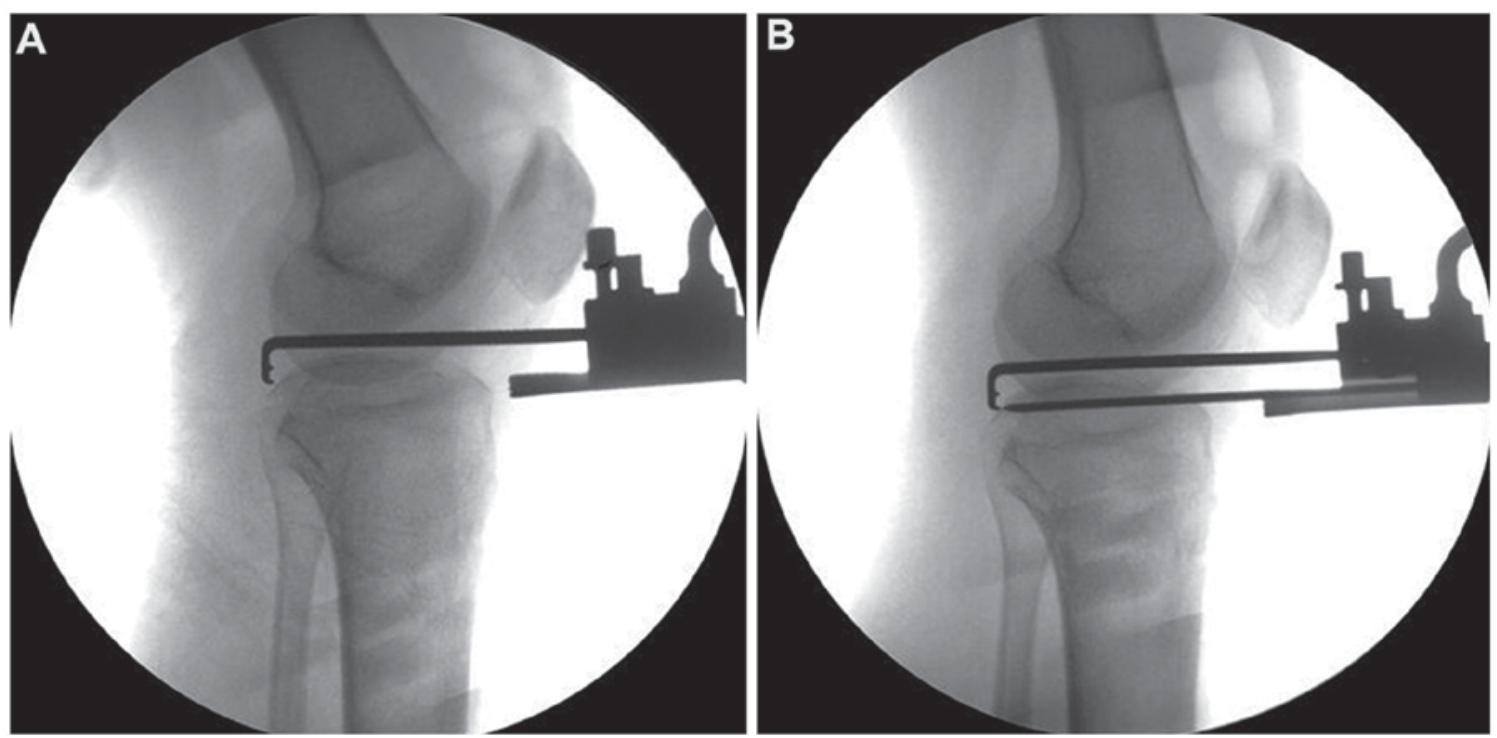

Figure 7. Positioning of the guide plate and guide needle. A C-arm fluoroscope was used to determine (A) the position and direction of the guide plate and (B) the position, direction and depth of the guide needle during needle insertion.

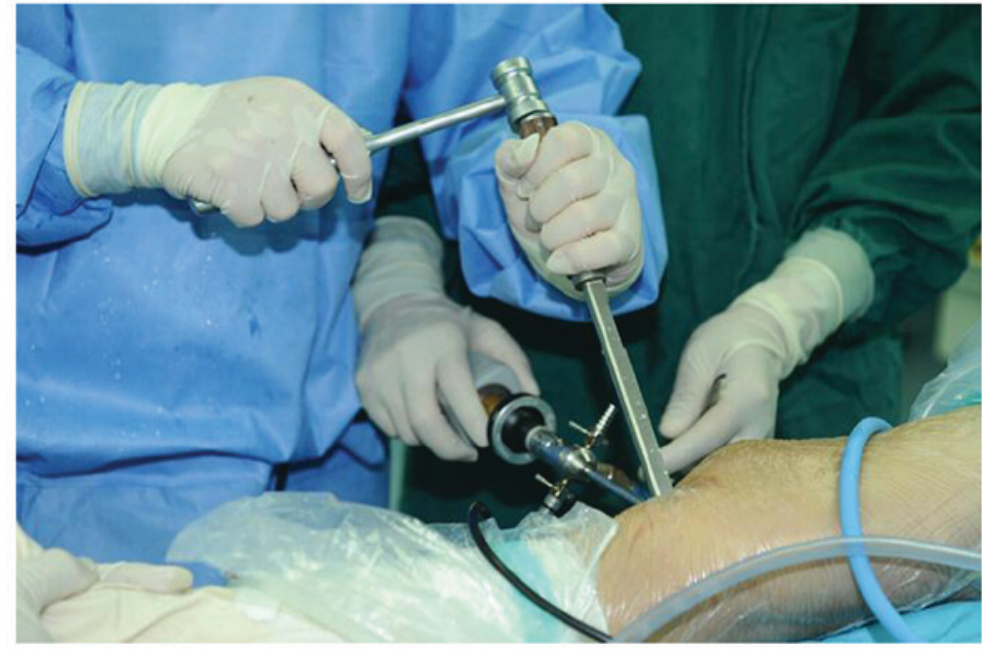

Figure 8. Construction of the bone trough. After the insertion of the guide needle, a $9 \mathrm{~mm}$ hollow drill was inserted along with the guide needle. The depth of the hollow drill was $5 \mathrm{~mm}$ shorter than the depth of the guide needle. A $7 \mathrm{~mm}$ piston rod was placed in the $9 \mathrm{~mm}$ bone tunnel. A trough bone knife with an outer diameter of $9 \mathrm{~mm}$ was inserted along with the piston rod, and the broken bone fragments were removed. A bone trough of the tibial plateau with a 9-mm diameter and width was constructed.

Implantation of meniscal allograft. The allogenic meniscus was placed into the knee joint through the frontal entrance of the arthroscope. The anterior and posterior $1 / 3$ of the traction line was used to properly place the meniscus (Fig. 9B). Varus and valgus knee alignments contributed to the placement of the meniscus. The meniscal bone bridge was inserted into the bone trough of the tibial plateau, and the position of the anterior and posterior horns of the meniscus was adjusted by altering the anterior and posterior positions of the bone bridge. After the insertion of the allogenic meniscus, it was verified whether the position of the femoral condyle was compatible with that of the meniscus to allow for a certain degree of knee joint flexion. The bone bridge of the meniscus was gently pressed and knocked. The knee joint was straightened to make the bone plate gently press the bone bridge to insert it into the bone trough of the tibial bone. The two ends of the traction line at the anterior and posterior horns of the meniscus were knotted and fixed in the medial tibial tuberosity.

Postoperative treatment. Routine irrigation of the joint cavity and closure of the wound, compress dressing and cold compress were performed. Long contraction exercises of the quadriceps were performed immediately after the surgery. The knee joint was straightened at $0^{\circ}$ by using a knee brace. At postoperative day 3 , knee joint exercises were performed. At 3 weeks after surgery, the knee joint was fully straightened and the degree of flexion was within $60^{\circ}$. At 6 weeks after surgery, the degree of knee flexion was increased by $10^{\circ}$ weekly and gradually exceeded $90^{\circ}$. At 12 weeks after surgery, the knee joint activity was restored to normal levels. At 24 weeks after surgery, patients could walk freely and independently. At 36 weeks after surgery, 

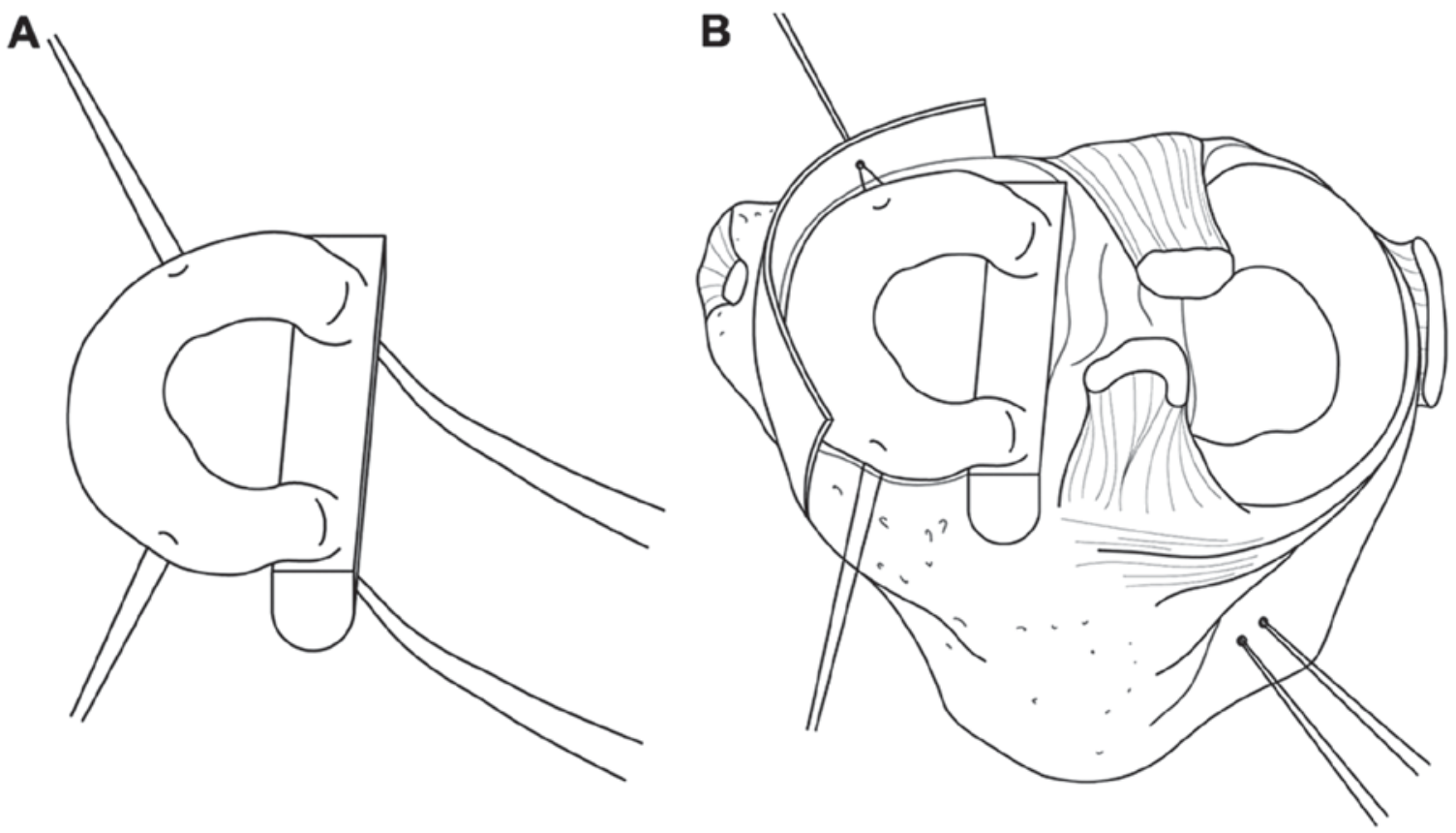

Figure 9. Placement of the meniscal allograft into the knee joint cavity. (A) The traction line in the anterior and posterior horns of the meniscus passed the anterior medial tibia through the hole. (B) Anterior and posterior 1/3 of the traction line was used to properly place the meniscus.

patients were allowed to run and ride a bicycle. At 48 weeks after surgery, patients could perform sports activities.

Follow-up and evaluation index. Knee function was assessed before and after surgery using the visual analog scale (VAS), Lysholm score and International Knee Documentation Committee (IKDC) grading (17). All patients were assessed for 6-28 months following surgery. As previously described by Mink (18) and Fischer (19), the meniscus magnetic resonance imaging (MRI) evaluation criteria were: 0 is a uniform low-intensity signal of the normal meniscus; Grade I injury is spherical or irregular punctate high-intensity signal which does not extend to the joint surface; Grade II injury is horizontal line high-intensity signal which does not affect the joint surface but extends to the junction of the meniscus joint capsule; Grade III injury is abnormally high-intensity signal extending to the meniscal joint surface; Grade IV injury is meniscal lesions or fragments. MRI evaluation of articular cartilage injury was based on Recht's criteria (7). Three patients underwent surgery for other reasons at 3,9 and 18 months after surgery.

Statistical analysis. SPSS 12.0 statistical software (SPSS, Inc., Chicago, IL, USA) was used for data analysis. Measurement data were presented as the mean \pm standard deviation. The degree of knee flexion VAS score and Lysholm score were statistically compared using a paired t-test, before and after postoperative follow-up. Wilcoxon signed-rank test was adopted to compare IKDC grades and cartilage injury. $\mathrm{P}<0.05$ was considered to indicate a statistically significant difference.

\section{Results}

Baseline data. From November 2006 to July 2009, 21 patients (14 males and 7 females; age range, 19-49 years; mean age, 31.6 years) with meniscal resection (22 cases; one patient required meniscal resection on both knees) underwent allogeneic meniscus transplantation. There were 16 cases with lateral meniscal injury and 6 cases with medial meniscal injury. The time interval from meniscal resection to allogenic meniscal transplantation was 0-9 years, (mean, 3.8 years). Knee MRI examination demonstrated no articular cartilage lesions or callus formation in 12 cases, 8 cases with interstitial pili spinous hyperplasia had a small amount of osteophyte formation at the edge of the tibial plateau. Arthroscopic evaluation of articular cartilage injury with modified Outerbridge classification (20) was performed: Grade 0 in 12 patients, grade I in 5 patients, grade II in 3 patients and grade III in 1 patients The blood loss was $49.2 \pm 10.7 \mathrm{ml}$, operative length was $1.4 \pm 0.4 \mathrm{~h}$ and the length of hospitalization was $14.7 \pm 2.1$ days.

Knee joint function. All patients were followed up for 6-28 months (mean, 16.3 months). No surgical injuries, infections or requirements to remove the transplanted meniscus occurred. No abnormalities were noted in the immune parameters after surgery, and no obvious allogeneic immune rejection was reported. The degree of knee joint motion was completely straightened before surgery. The degree of knee joint straightening was comparable before and after surgery. The degree of knee flexion was $105-150^{\circ}$ (mean, $121.4^{\circ}$ ) before surgery and $120-150^{\circ}$ (mean, $132.7^{\circ}$ ) at the final follow-up, there was no statistical significance between the degree of knee flexation $(t=1.123, P=0.268)$. Two patients neglected rehabilitation exercises after surgery. During the follow-up, the degree of knee flexion $\left(120^{\circ}, 120^{\circ}\right)$ was not restored to the preoperative level $\left(135^{\circ}, 150^{\circ}\right)$. One patient had limited knee flexion $\left(105^{\circ}\right)$ preoperatively and improved to $135^{\circ}$ after postoperative exercise. All patients could walk during follow-up. VAS scores, Lysholm scores and IKDC classification results are presented in Table I. Prior to surgery, patients with normal and almost normal IKDC classification (Grade A and B) accounted for $14.3 \%$, which was 
significantly increased to $76.2 \%$ postoperatively $(\mathrm{P}=0.0003)$. The VAS score significantly decreased while Lysholm score significantly increased in the final follow-up compared with before operation (Table I).

MRI performance. MRI examinations were performed prior to surgery in all patients. All MRI images indicated grade III or above meniscal injury signal. At 1 year after surgery, 13 cases were examined by MRI. One patient presented with grade I and II signal at the anterior horn of the transplanted meniscus and two patients presented with grade I and II signal at the posterior horn, but no clinical symptoms were documented. The remaining meniscal allografts were well-shaped, whereas uneven internal signal was noted. Of the 13 patients receiving preoperative MRI examination, 6 cases had grade 0 articular cartilage, 4 cases had grade I, 2 cases had grade II and 1 case had grade III. At 1 year after surgery, 5 cases had grade 0, 4 cases had grade I, 3 cases had grade II and 1 case had grade III. No statistical significance in the grade of the meniscal injury signal was observed before or after surgery $(\mathrm{Hc}=0.570, \mathrm{P}=0.966)$.

Arthroscopic observation. In the current study, 1 patient received arthroscopy at 3 months after medial meniscus transplantation. The edge of the meniscus and the joint capsule were almost healed and synovial membrane hyperemia was noted at the periphery. The meniscus was intact in morphology with a sharp edge, poor stability and brittle texture. The articular cartilage was evaluated as Outerbridge grade II before and after surgery. At 9 months after the lateral meniscus transplantation, arthroscopy revealed that the transplanted meniscus and joint capsule were properly healed, and the anterior and posterior horns were fixed firmly. The meniscus was intact in shape, the texture became hard and the edge was slightly blunt The articular cartilage was assessed as Outerbridge grade I before and after operation. At 18 months after lateral meniscus transplantation, arthroscopy demonstrated that the meniscus graft and joint capsule were well healed, and the appearance and texture were similar to the normal meniscus. The margin was slightly rough and then trimmed under arthroscopy (Fig. 10). The articular cartilage was evaluated as Outerbridge grade 0 before and after operation.

\section{Discussion}

Following meniscal allograft transplantation, the symptoms of pain, swelling and knee joint function can be significantly improved $(21,22)$. However, it is challenging to perform arthroscopic and minimally invasive meniscus transplantation due to the lack of necessary special surgical instruments and standard procedures. In the current study, a minimally invasive surgical approach was established and self-designed surgical instruments were used to successfully accomplish meniscal allograft transplantation.

One of the most vital steps in meniscal transplantation is to fix the anterior and posterior horns of the meniscus at a precise anatomical position (9). The meniscal graft can function properly when implanted to the original anatomical position. Intraoperative anteroposterior horns can be fixed by two approaches, including direct suturing of the soft tissues

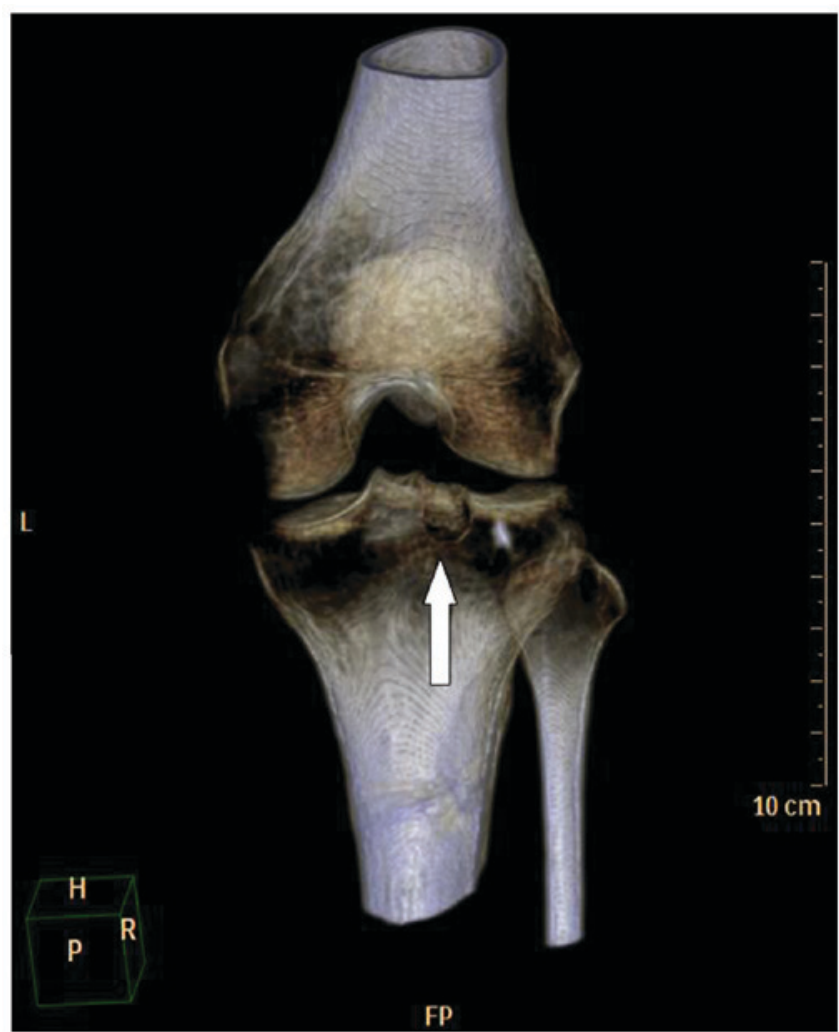

Figure 10. Computed tomography image of the cross-sectional status of the bone bridge and bone trough. Arrow indicates the margin of the meniscus.

of the anterior and posterior horns of the meniscus, and bone fixation of the anterior and posterior horns with bone anchors. A previous cadaveric study (11) demonstrated that lateral meniscus transplantation without a good anterior-posterior angulation will yield poor function similar to meniscal resection.

Following meniscal allograft transplantation without the use of bone anchor fixation, extensive cartilage degeneration will occur in the knee weight-bearing area (23). Alhalki et al (24) compared the contact mechanics of three fixation techniques of the meniscal anterior and posterior horn, including direct suturing, bone screw fixation, and a combination of the two. It was demonstrated that bone screw fixation was closest to the contact mechanics of the normal knee joint, and the combined use of two methods did not enhance the quality of meniscal fixation. These results indicated that meniscus transplantation requires anterior and posterior horn fixation, and that the biomechanical property of bone fixation is superior to that of the soft tissue suturing method (22-25). Bone fixation is currently the gold standard for meniscal allograft transplantation (13).

The meniscus anterior and posterior skeletal fixation methods include two techniques. First, the anterior and posterior horns of the meniscus are made into two bone plugs and implanted into the bone tunnels of the anterior and posterior horns of the tibial plateau. Second, a bone bridge between the anterior and posterior horns of the meniscus is maintained and implanted into the bone trough between the anterior and posterior horns of the meniscus of the tibial plateau. This can maintain the normal distance and anatomic 
relationship between the anterior and posterior horns of the native meniscus (1) and can be applied in medial or lateral meniscus transplantation. Because the anterior and posterior horns of the lateral meniscus are closer (1 cm or less) and there is no structure between the two horns, this technique is more applicable to lateral meniscus transplantation. The main problem is the lack of standard surgical instruments. Surgeons can only cut and grind bones manually to construct the bone trough and bridge. Without standardized instruments for measurement and cutting, it is difficult to ensure that the shape and size of the bone trough and bridge can be properly matched $(8,15)$. The mismatch of shape and size between bone bridge and bone trough reduces the accuracy of surgery, and makes it difficult to achieve optimal stability of the anterior and posterior horns of the meniscus. Intraoperative graft construction is time-consuming and prolonged surgery increases the risk of surgery. Bone bridge implantation is an open surgery or small incision surgery rather than arthroscopic minimally invasive surgery (8). To accomplish the arthroscopic minimally invasive surgery, an arthroscopic and minimally invasive meniscal graft transplantation and corresponding surgical instruments were designed in the current study, which simplify the surgical procedures, increase surgical accuracy, enhance the stability of the bone bridge and bone trough and yield favorable outcomes. During bone bridge implantation, the cross-sectional shape of the anterior and posterior horns of the bone bridge and tibial plateau must be matched, which can be made into 'keyhole', 'dovetail' or trough shapes. 'Keyhole' and 'dovetail' shapes can be applied in open surgery and 'small incision' surgery in which the bone bridge can be inserted into the bone trough (8). Only the trough shape can be applied in arthroscopic minimally invasive surgery, as it is more convenient, safe and causes minimal trauma. Therefore, the bone bridge in trough method was used in this study.

The placement of the guide plate is a critical step in the surgical procedure. To obtain accurate medial and lateral position of the meniscus in the coronal position, the guide plate must be positioned above the anterior and posterior horns of the original meniscus. The guide plate should be inclined at an angle consistent with the inclination of the tibial plateau surface. The precise depth of the bone trough depends on whether the guide plate adheres to the peak of the tibial plateau. C-arm fluoroscopy can be performed to verify the position of the guide plate and needle when necessary.

The location of the meniscal allograft should be identical that of the original meniscus and adapted to the shape of the femoral condyle to deliver stress on the optimal area. It is necessary to ensure that the position of the bone trough is accurately determined. The positions of the traction wires on the transplanted meniscus are the same as those of the bone tunnel. After the insertion of the allogenic meniscus, the knee joint is allowed to move in a certain range of flexion and extension activities to verify whether the position of the femoral condyle is compatible with the position of the meniscus. The position where the least motion of the meniscus is obtained is the optimal position.

One of the key problems that can arise after allogeneic meniscal transplantation is extrusion of the allograft, with an incidence up to $87 \%$ (12). In the current study, a 'four-point fixation' technique was adopted, in which the anterior horn, posterior horn, the junction of body of the meniscus and anterior horn and the junction of body and the posterior horn were fixed to the corresponding anatomical positions on the tibial plateau, which effectively prevented and reduced the risk of extruded allograft.

The present study had certain limitations. It was a single center study with small sample size and was retrospective. Further prospective, multicenter studies with larger sample sizes are required to verify the current findings.

Intraoperatively, the sutures at the junction between the body and the anterior and posterior horns were relatively loose. During the knee flexion and extension activities, the meniscus graft could simulate the function of the normal meniscus. In the current study, the bone suturing technique was selected because alternative approaches, such as interface nails, would be likely to lead to bone bridge fracture or injury to the anterior horn of the meniscus.

In conclusion, the surgical techniques and instruments used in this study could be used to standardize meniscal graft transplantation and avoid the incidence of surgical errors caused by mismatched size and shape of the bone bridge and bone trough. The four-point fixation of the tibial plateau facilitates and enhances the stability of the meniscal allograft, reducing the risk of meniscal extrusion, which ensures the postoperative recovery of meniscal function.

\section{Acknowledgements}

Not applicable.

\section{Funding}

This study was funded by the Key Research Project for Health Development of Beijing Municipal Health and Family Planning Commission (grant no. 2018-1-5021).

\section{Availability of data and materials}

The datasets used and/or analyzed during the present study are available from the corresponding author on reasonable request.

\section{Authors' contributions}

YaZ, SH, LL, HZ, YiZ and DL designed the study and performed the operation. LC, YD and DG collected and analyzed the data. HJ performed literature research, collected patient information and prepared the manuscript. All authors read and approved the final manuscript.

\section{Ethics approval and consent to participate}

The current study was approved by the Ethics Committee of the First Affiliated Hospital of PLA General Hospital (304 Hospital; Beijing, China).

\section{Patient consent for publication}

All participants provided written informed consent for publication. 


\section{Competing interests}

The authors declare that they have no competing interests.

\section{References}

1. Lee AS, Kang RW, Kroin E, Verma NN and Cole BJ: Allograft meniscus transplantation. Sports Med Arthrosc Rev 20: 106-114, 2012.

2. Zhang YD, Hou SX, Zhang YC, Luo DZ, Zhong HB and Zhang H: Arthroscopic combined medial and lateral meniscus transplantation after double-tunnel, double-bundle anterior cruciate ligament reconstruction in the same knee. Knee 19: 953-958, 2012

3. Elattar M, Dhollander A, Verdonk R, Almqvist KF and Verdonk P: Twenty-six years of meniscal allograft transplantation: Is it still experimental? A meta-analysis of 44 trials. Knee Surg Sports Traumatol Arthrosc 19: 147-157, 2011.

4. Hergan D, Thut D, Sherman O and Day MS: Meniscal allograft transplantation. Arthroscopy 27: 101-112, 2011.

5. Milachowski KA, Weismeier K and Wirth CJ: Homologous meniscus transplantation: Experimental and clinical results. Int Orthop 13: 1-11, 1989.

6. Sohn DH and Toth AP: Meniscus transplantation: Current concepts. J Knee Surg 21: 163-172, 2008

7. Cole BJ, Carter TR and Rodeo SA: Allograft meniscal transplantation: Background, techniques, and results. Instr Course Lect 52: 383-396, 2003.

8. Farr J and Gersoff W: Current meniscal allograft transplantation. Sports Med Arthrosc Rev 12: 69-82, 2004.

9. Kelly BT, Brophy RH and Rodeo S: Meniscal allograft transplantation: Surgical technique. Techniques Knee Surg 3: 8-18, 2004.

10. Abat F, Gelber PE, Erquicia JI, Pelfort X, Gonzalez-Lucena G and Monllau JC: Suture-only fixation technique leads to a higher degree of extrusion than bony fixation in meniscal allograft transplantation. Am J Sports Med 40: 1591-1596, 2012.

11. Chen MI, Branch TP and Hutton WC: Is it important to secure the horns during lateral meniscal transplantation? A cadaveric study. Arthroscopy 12: 174-181, 1996.

12. Paletta GA Jr, Manning T, Snell E, Parker R and Bergfeld J: The effect of allograft meniscal replacement on intraarticular contact area and pressures in the human knee. A biomechanical study. Am J Sports Med 25: 692-698, 1997.

13. Shelton WR and Dukes AD: Meniscus replacement with bone anchors: A surgical technique. Arthroscopy 10: 324-327, 1994.
14. Sekaran SV, Hull ML and Howell SM: Nonanatomic location of the posterior horn of a medial meniscal autograft implanted in a cadaveric knee adversely affects the pressure distribution on the tibial plateau. Am J Sports Med 30: 74-82, 2002.

15. Wirth CJ, Peters G, Milachowski KA, Weismeierz KG and Kohn D: Long-term results of meniscal allograft transplantation. Am J Sports Med 30: 174-181, 2002.

16. AATB: Standards for tissue banking. American Association of Tissue Banks. 10th edition. McLean, Vigina, USA, 2002.

17. Ibarra C, Izaguirre A, Villalobos E, Masri M, Lombardero G, Martinez V, Velasquillo C, Meza AO, Guevara V and Ibarra LG: Follow-up of a new arthroscopic technique for implantation of matrix-encapsulated autologous chondrocytes in the knee. Arthroscopy 30: 715-723, 2014.

18. Mink JH, Levy T and Crues JV III: Tears of the anterior cruciate ligament and menisci of the knee: MR imaging evaluation. Radiology 167: 769-774, 1988.

19. Fischer SP, Fox JM, Del Pizzo W, Friedman MJ, Snyder SJ and Ferkel RD: Accuracy of diagnoses from magnetic resonance imaging of the knee. A multi-center analysis of one thousand and fourteen patients. J Bone Joint Surg Am 73: 2-10, 1991

20. Slattery $C$ and Kweon CY: Classifications in brief: Outerbridge classification of chondral lesions. Clin Orthop Relat Res 476: 2101-2104, 2018.

21. LaPrade RF, Wills NJ, Spiridonov SI and Perkinson S: A prospective outcomes study of meniscal allograft transplantation. Am J Sports Med 38: 1804-1812, 2010.

22. Stone KR, Adelson WS, Pelsis JR, Walgenbach AW and Turek TJ: Long-term survival of concurrent meniscus allograft transplantation and repair of the articular cartilage: A prospective two- to 12-year follow-up report. J Bone Joint Surg Br 92: 941-948, 2010.

23. Lazović D, Wirth CJ, Knösel T, Gossé F and Maschek HG: Meniscus replacement using incongruent transplants-an experimental study. Z Orthop Ihre Grenzgeb 135: 131-137, 1997 (In German)

24. Alhalki MM, Howell SM and Hull ML: How three methods for fixing a medial meniscal autograft affect tibial contact mechanics. Am J Sports Med 27: 320-328, 1999.

25. Marcacci M,Zaffagnini S, Marcheggiani Muccioli GM, Grassi A, Bonanzinga T, Nitri M, Bondi A, Molinari M and Rimondi E: Meniscal allograft transplantation without bone plugs: A 3-year minimum follow-up study. Am J Sports Med 40: 395-403, 2012.

This work is licensed under a Creative Commons Attribution-NonCommercial-NoDerivatives 4.0 International (CC BY-NC-ND 4.0) License. 